\title{
Review on Colonrectal Cancer
}

\author{
Xinghan Huang ${ }^{1}$ \\ ${ }^{1} 11^{\text {th }}$ grade student, 101, unit 4, building 15th, No.8th yard, Hua mao cheng, Chaoyang District, Beijing
}

\begin{abstract}
The aim of the study of colon cancer is to introduce the basic information of cancer cell and the basic information of colon cancer. The research on colon cancer gave the information about hallmarks of cancer cell. exaggerated continuous proliferation, resistance to cell death as well as tumor suppressors, angiogenesis, spreading and metastasis and replicative immortality, the symptoms of colon cancer: Frequent bowel dysfunction (Diarrhea-constipation), Abdominal pain, nausea \& vomiting, Pain during defecation, and Bloating, the types of colon cancer: Familial Adenomatous Polyposis (FAP) and Lynch syndrome, nonpolyposis Colorectal Cancer, and the treatment of colon cancer: Treatment strategies differ according to the stage and development of the tumor. Surgery and adjuvant chemo are the two common methods in stages 2 and 3 of the disease. Palliative care is most common in patients at stage 4 to improve quality of life. The information which extracted from papers from other researchers formed the research of colon cancer.
\end{abstract}

\section{Introduction of cancer}

Cancer is a disease caused by uncontrolled cell division (proliferation). The risk factors for developing cancer could be any of the following: UV rays, gamma rays, radioactive chemicals, and nuclear wastes. Uncontrolled cell division is caused by the DNA damage, which (causes DNA mutations that can then lead to) dysfunction of tumor suppressors and oncogenes. Exaggerated cell proliferation is a characteristic feature in all cancer cells, although the histopathological form of the cell is normal (Weinberg 2014; Hall et al. 1993). Cancer cells are mainly governed by six hallmarks: exaggerated continuous proliferation, resistance to cell death as well as tumor suppressors, angiogenesis, spreading and metastasis, and replicative immortality. (Hanahan and Weinberg 2000). Here, we will review the six hallmarks of cancer before we delve slightly into colon cancer as one of the fatal tumors. The first hallmark, according to Hanahan and Weinberg, is sustaining proliferative signaling. Proliferative signaling is usually sustained in malignant tumors due to the accidental introduction of mutations into one or more of the proto-oncogenes, which by then becomes an oncogene. Oncogenes are a kind of gene that promotes cell growth and proliferation, so when overactive, these can cause aberrant patterns of cell growth. For example, the Ras family are all considered as proto-oncogenes, as they control cell growth. Therefore, mutated Ras is one of the most common markers in cancer as mutations can cause them to lose the ability to regain inactivity (trapped in an "always-on" state) (Cox and Der 2010; DeCaprio 2009; Shih et al. 1979). Mutations might occur as a consequence of DNA damage, which can then turn proto-oncogene into oncogenes, causing uncontrolled cell growth. The unlimited cell growth provides a good environment to develop cancer because the increased number of cell division cycles for the cell increases the chances for more mutations to take place in other positions, leading to the development of the rest of cancer hallmarks. The second hallmark is evading growth suppressors. Tumor suppressors are cell cycleregulating proteins that aim to limit and control the cell cycle at different checkpoints. P53 and p27 are examples of tumor suppressors (Francis et al., 2013). P53 is one of the main tumor suppressors that works at G1 checkpoint. P53 suppresses the process of cell growth when it detects DNA damages or other kinds of mutations by halting cell cycle at this point. P53 can then trigger a pathway to fix the detected DNA damage. If this is proven unsuccessful, P53 then plays its third role by triggering apoptosis. The third hallmark is resisting cell death. There are three types of cell death mechanisms: apoptosis, autophagy, and necrosis. In this review, the author will focus more on apoptosis and cancer. Apoptosis is triggered upon cell damage, during cell development, upon detachment from the extracellular matrix, or many other reasons. Apoptosis can be activated by different intracellular or extracellular signaling pathways. Cancer cells can evade programmed cell death (apoptosis) by loss-of-function mutations in p53 gene, promoting anti-apoptotic, and ignoring proapoptotic signals (Hanahan and Weinberg 2000; Miyashita et al. 1994; Hall et al. 1993). The fourth hallmark is enabling replicative immortality. This hallmark is a result of the reactivation of telomerases, which are enzymes that extend telomers. Telomers are caps made of DNA that flank chromosomes and function as protective shields against wearing out during cell division cycles. Telomers, however, wear out (get shortened) as we age, and this is thought to be one of the main factors behind aging. Telomerases are active in

\footnotetext{
* Corresponding author: 1798502804@qq.com
} 
germlines and certain hematopoietic but inactive in somatic cells. Therefore, reactivation of telomerase can cause "immortal" telomers, leading to nearly "immortal" cells (Shammas 2011). As a result, cancer cells are equipped against aging and hence are able to keep the integrality of mutated genes. As mentioned before, increased half-life and number of cell cycles is the recipe for higher chances of new mutations being introduced (Weinberg 2014). The fifth hallmark is inducing angiogenesis. Inducing angiogenesis will aid cancer cells to form a new network of blood vessels around the tumor.
This provides cancer cells with more energy and Oxygen needed for its increased cell division cycles. The last hallmark is activating invasion and metastasis. This gives cancer cells the ability to transport to other organs/locations in the body. This will cause forming new tumors in different places that have the properties of the original tumor. As a result, this leads to fatal consequences as new tumors forming in different organs interfere with the daily functions of these organs (Hanahan and Weinberg 2000).

Estimated number of prevalent cases (5-year) worldwide, both sexes, all ages

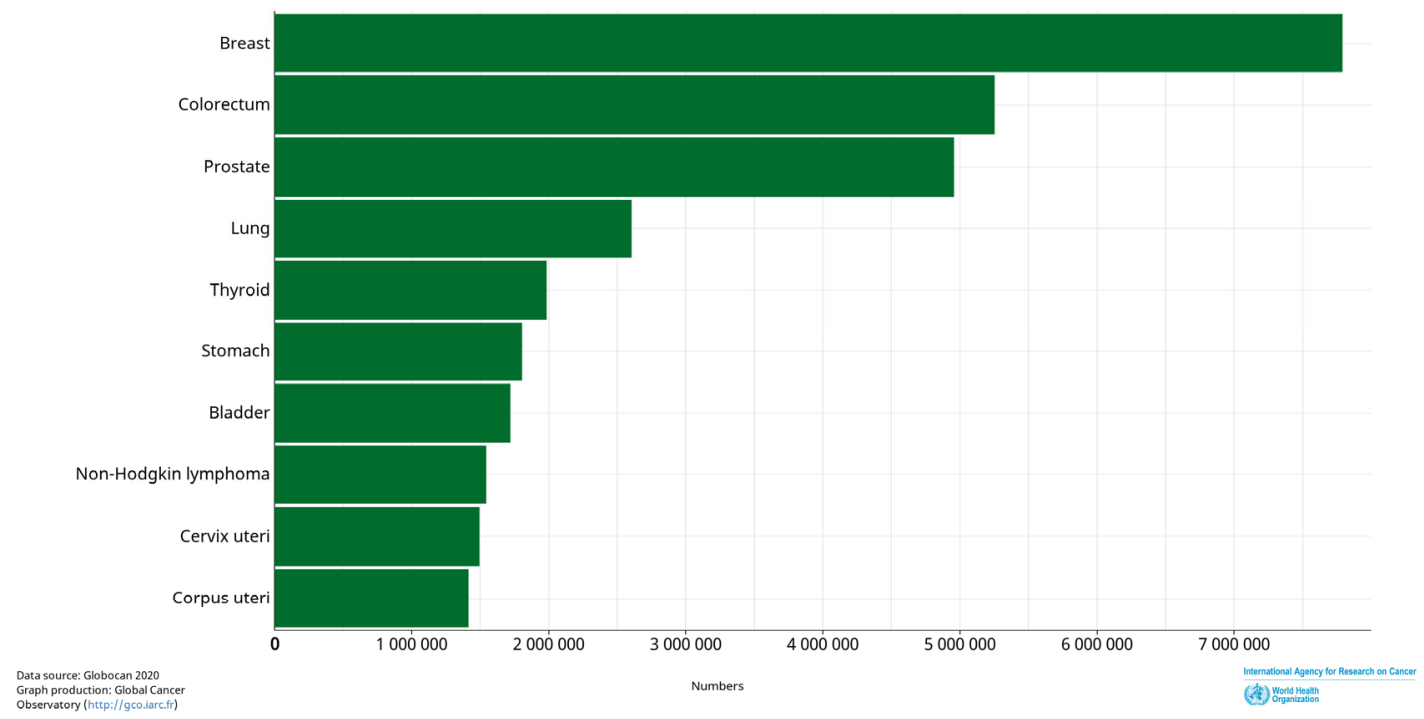

Fig. 1. Estimated number of incident cases and deaths worldwide, both sexes, all ages(reproduced from http://globocan.iarc.fr)

Estimated number of prevalent cases (5-year) China, both sexes, all ages

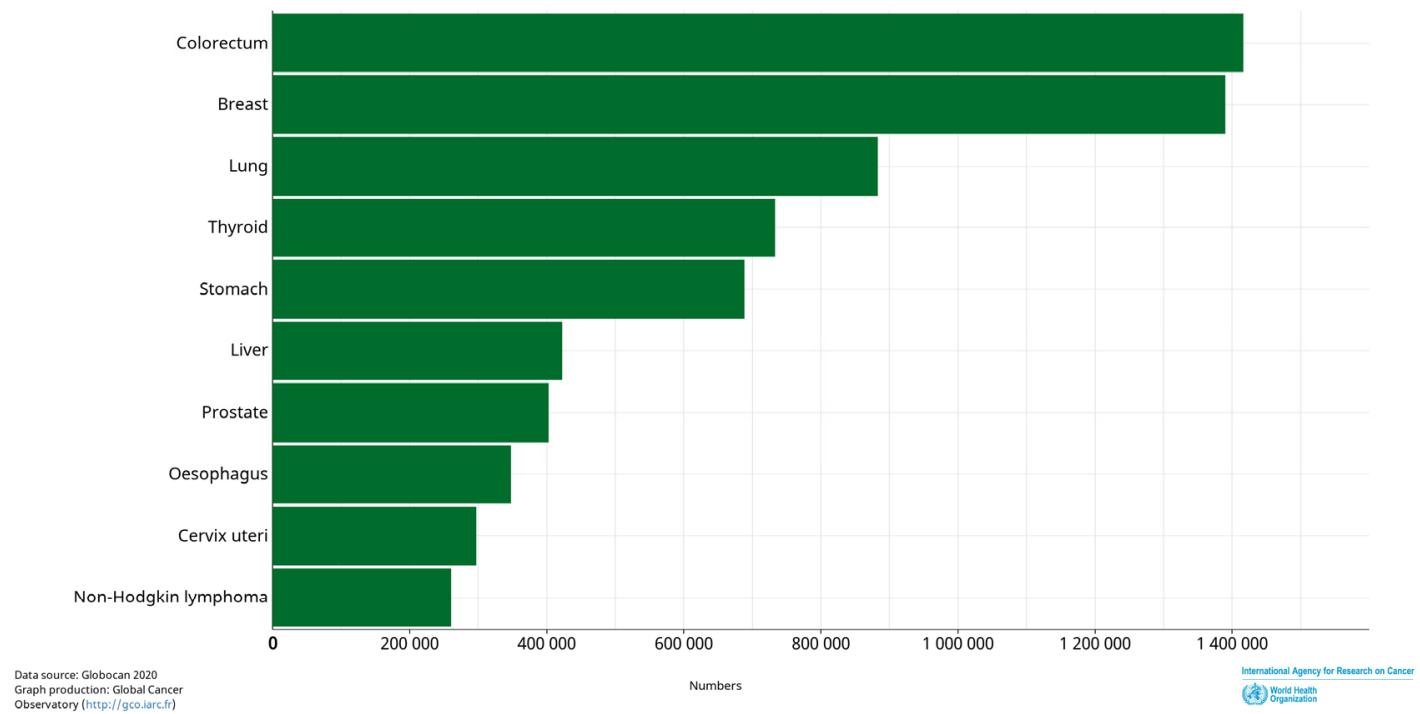

Fig. 2. Prevalence in numbers, China, both sexes.png(reproduced from http://globocan.iarc.fr)

\section{Colon cancer}

One of the fatal types of cancer is colon cancer. Before we delve into it, let us have an overview of the anatomy of the colon. The large intestine is a part of the digestive system. The major functions of the large intestine are absorbing water and minerals from the remaining indigestible food matter, in addition to transmitting and releasing waste material out of the body. The colon is the 
main part of the large intestine. The colon consists of four parts: ascending colon, transverse colon, descending colon, and sigmoid colon. Ascending colon locates at the right side of the abdominal cavity to the level of the liver. The transverse colon locates at hepatic flexure and continues across the abdominal cavity. Descending colon locates at the splenic flexure and moves downward along the abdominal cavity. The sigmoid colon locates at the pelvis and joins the rectum at the rectosigmoid junction. Cancer cells are more likely to form in these parts of the colon in different percentages: ascending colon for $5 \%$, caecum for $15 \%$, transverse colon and flexures for $10 \%$, descending colon for $3 \%$, sigmoid colon for $29 \%$, and rectum for $38 \%$.
$\mathrm{CRC}$, the full spelling is colorectal cancer, is one of the common types of cancer occurring in males and females. Colon cancer is considered as one of the deadliest types, as $25 \%$ of patients will develop metastases during the course of the disease (Total metastatic is almost 50\%) (Májek 2012; Lee GH 2015; Li FY 2009).

The number of incidence cases on a global scale is around 5,500,000.

The number of incidence cases in China is around $1,400,000$ $25 \%$

The rate of incidence cases for worldwide is around

The rate of mortality cases for worldwide is around $12.5 \%$

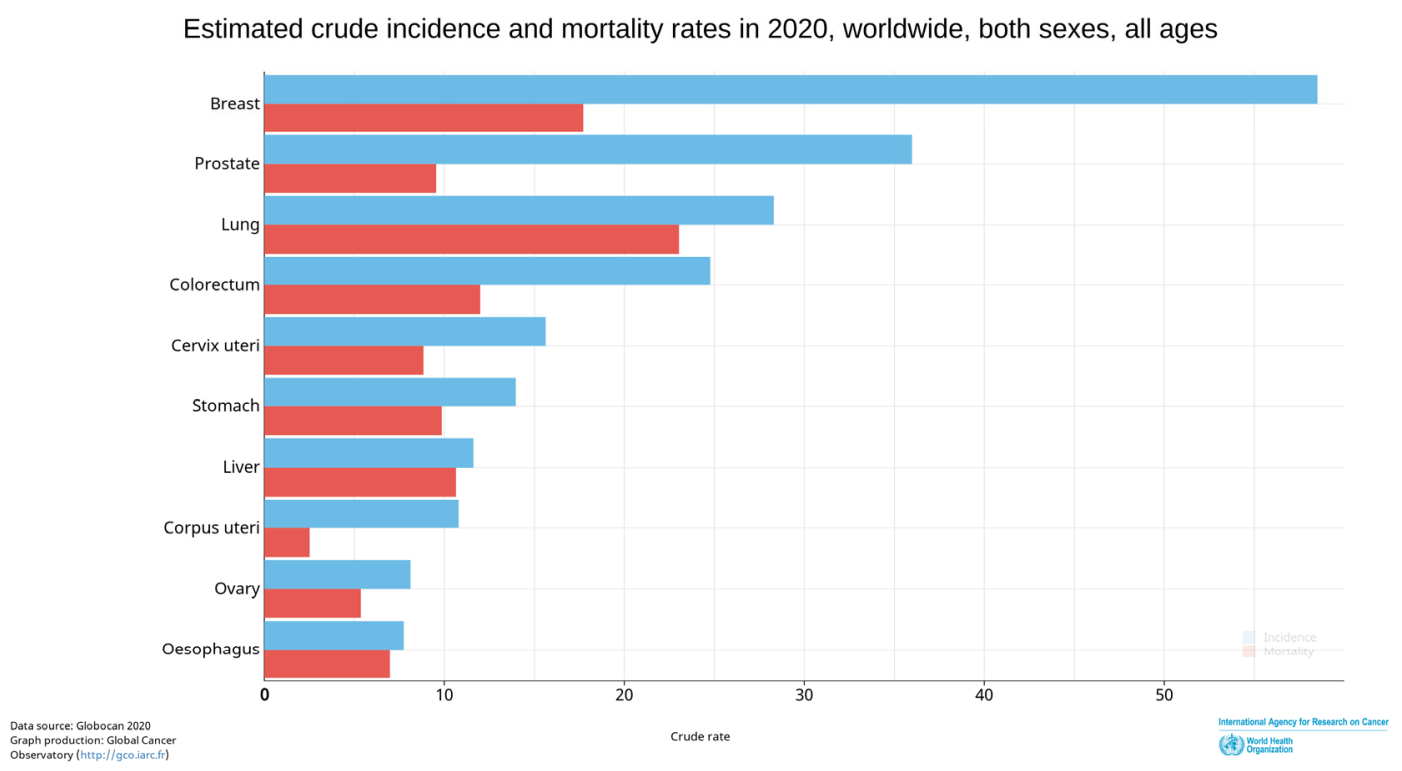

Fig. 3. Incidence and Death in numbers, World, both sexes.png(reproduced from http://globocan.iarc.fr)

In terms of diagnosis, KRAS, NRAS, and BRAF are three biomarkers used for detection, diagnosis, and treatment selection for CRC. KRAS is the most common biomarker detected in CRC, especially KRAS exon 2 mutation ( $40 \%$ of CRC patients). BRAF is detected in $12-14 \%$ of CRC patients, and it is more common in right side colon tumors. NRAS is detected in $5 \%$ of CRC patients (Schwartzberg 2014; Shepherd 2013). The common symptoms of CRC: Frequent bowel dysfunction (Diarrhea-constipation), Abdominal pain, nausea \& vomiting, Pain during defecation, and Bloating. The common signs of CRC: Haematochezia (blood with stool), weight loss, and anemia, and hence fatigue. Hereditary CRC comprises $\sim 6 \%$ of all CRC cases. There are two types of hereditary CRC: Familial Adenomatous Polyposis (FAP) and Lynch syndrome, which is previously known as Hereditary Non-polyposis Colorectal Cancer (HNPCC). FAP comprises $1 \%$ cases of all CRC cases. FAP is caused by mutation of Adynomatous polyposis Coli gene (APC, tumor suppression gene). Hundreds of polyps are typically found in FAP patients. HNPCC comprises $5 \%$ cases of all CRC cases. HNPCC is caused by mutation of DNA mismatch repair gene (hMSH1, hMSH2). The development of CRC can be tracked in a stage-oriented fashion to aid diagnosis and determination of treatment strategy. CRC develops over 4 stages with a decreasing survival rate on average, that is, $93 \%$ in stage 1 , around $85 \%-72 \%$ in stage 2 , around $83 \%-44 \%$ in stage 3 , and $8 \%$ in stage 4 (Siegel 2017; Siegel 2014; Markowitz 2009; Fearon 2011; Galiatsatos 2006). Survived patients are the patients who suffered from CRC and survived for five years after total cure. In the first stage, the tumor invades mucosa. In the second stage, the tumor invades Sub-serosa and penetrates the surface of the peritoneum. In the third stage, the tumor settles in sub-serosa. In the fourth stage, the tumor metastasizes to different organ/s. Treatment strategies differ according to the stage and development of the tumor. Surgery and adjuvant chemo are the two common methods in stages 2 and 3 of the disease. Palliative care is most common in patients at stage 4 to improve quality of life (Markowitz 2009; Fearon 2011; Galiatsatos 2006).

\section{References}

1. Cox AD \& Der CJ 2010 Ras history: The saga continues. Small GTPases 1 2-27. 
2. DeCaprio JA 2009 How the Rb tumor suppressor structure and function was revealed by the study of Adenovirus and SV40. Virology 384 274-284

3. Hall, P.A., McKee, P., Menage, H.D., Dover, R. and Lane, D.P., 1993. High levels of p53 protein in UVirradiated normal human skin. Oncogene, 8(1), pp.203-207.

4. Hanahan D \& Weinberg RA 2000 The hallmarks of cancer. Cell 100 57-70.

5. Lee, G.H., Malietzis, G., Askari, A., Bernardo, D., Al-Hassi, H.O. and Clark, S.K., 2015. Is right-sided colon cancer different to left-sided colorectal cancer? -a systematic review. European Journal of Surgical Oncology (EJSO), 41(3), pp.300-308.

6. Li, F.Y., 2009. Colorectal cancer, one entity or three. Journal of Zhejiang University Science B, 10(3), pp.219-229.

7. Májek, O., Gondos, A., Jansen, L., Emrich, K., Holleczek, B., Katalinic, A., Nennecke, A., Eberle, A. and Brenner, H., 2012. Survival from colorectal cancer in Germany in the early 21 st century. British journal of cancer, 106(11), pp.1875-1880.

8. Miyashita, T., Krajewski, S., Krajewska, M., Wang, H.G., Lin, H.K., Liebermann, D.A., Hoffman, B. and Reed, J.C., 1994. Tumor suppressor p53 is a regulator of bcl-2 and bax gene expression in vitro and in vivo. Oncogene, 9(6), pp.1799-1805.

9. Schwartzberg, L.S., Rivera, F., Karthaus, M., Fasola, G., Canon, J.L., Hecht, J.R., Yu, H., Oliner, K.S. and Go, W.Y., 2014. PEAK: a randomized, multicenter phase II study of panitumumab plus modified fluorouracil, leucovorin, and oxaliplatin (mFOLFOX6) or bevacizumab plus mFOLFOX6 in patients with previously untreated, unresectable, wild-type KRAS exon 2 metastatic colorectal cancer. Journal of clinical oncology, 32(21), pp.2240-2247.

10. Shammas, M.A., 2011. Telomeres, lifestyle, cancer, and aging. Current opinion in clinical nutrition and metabolic care, 14(1), p.28.

11. Shepherd, F.A., Domerg, C., Hainaut, P., Jänne, P.A., Pignon, J.P., Graziano, S., Douillard, J.Y., Brambilla, E., Le Chevalier, T., Seymour, L. and Bourredjem, A., 2013. Pooled analysis of the prognostic and predictive effects of KRAS mutation status and KRAS mutation subtype in early-stage resected non-small-cell lung cancer in four trials of adjuvant chemotherapy. Journal of clinical oncology, 31(17), p.2173.

12. Shih C, Shilo BZ, Goldfarb MP, Dannenberg A \& Weinberg RA 1979 Passage of phenotypes of chemically transformed cells via transfection of DNA and chromatin. Proc Natl Acad Sci U S A 76 5714-5718.

13. Siegel, R.L., Miller, K.D., Fedewa, S.A., Ahnen, D.J., Meester, R.G., Barzi, A. and Jemal, A., 2017. Colorectal cancer statistics, 2017. CA: a cancer journal for clinicians, 67(3), pp.177-193.
14. Markowitz, S.D. and Bertagnolli, M.M., 2009. Molecular basis of colorectal cancer. New England journal of medicine, 361(25), pp.2449-2460.

15. Fearon, E.R., 2011. Molecular genetics of colorectal cancer. Annual Review of Pathology: Mechanisms of Disease, 6, pp.479-507.

16. Galiatsatos, P. and Foulkes, W.D., 2006. Familial adenomatous polyposis. American journal of gastroenterology, 101(2), pp.385-398. 